\title{
ON THE LOCALIZATION OF THE SPECTRUM FOR SYSTEMS OF OPERATORS
}

\author{
ŞTEFAN FRUNZĂ
}

\begin{abstract}
Let $a=\left(a_{1}, \ldots, a_{n}\right)$ be a commuting system of linear continuous operators on a complex Banach space $X$. We show that, for any $x \in X$, the local analytic spectrum $\sigma(a, x)[1]$ is contained in the spectral hull of the local spectrum $\operatorname{sp}(a, x)[4]$.
\end{abstract}

Introduction. In this paper we shall define more accurately the relation between two notions of local spectrum for systems of operators.

Let $X$ be a complex Banach space and let $a=\left(a_{1}, \ldots, a_{n}\right)$ be a commuting system ( $n$-tuple) of linear continuous operators on $X$.

The simplest way to define the spectrum of an arbitrary element $x \in X$, with respect to $a$, is the following. Consider the union $\rho(a, x)$ of all open sets $D \subset C^{n}$ such that there exist $n$ analytic functions $f_{1}, \ldots, f_{n}: D \rightarrow X$, satisfying $\sum_{i=1}^{n}\left(z_{i}-a_{i}\right) f_{i}(z) \equiv x, z \in D$. Then the spectrum of $x$ with respect to $a$ is the set $\sigma(a, x)=C^{n} \backslash \rho(a, x)$. This notion of local spectrum was used in [1] for the study of spectral decompositions dependent on functional calculi.

In [4] the author has studied spectral decompositions not necessarily dependent on functional calculi and has found very useful to define another notion of local spectrum. Generally speaking, the spectrum of $x$ with respect to $a$ in our sense, denoted by $\operatorname{sp}(a, x)$, is the support of a certain differential form (or rather of a cohomology class of such forms). This notion was suggested by a new definition of the functional analytic calculus for several commuting operators, proposed in [7].

We have proved in [4] that $\operatorname{sp}(a, x) \subset \sigma(a, x)$ for any $x \in X$.

The main result of the present paper is that $\sigma(a, x)$ is contained in the spectral hull of $\operatorname{sp}(a, x)$, for any $x \in X$.

1. Preliminaries. We shall recall the definition of the Cauchy-Weil integral which will be our main tool in what follows (for details, see [7, §3] and [4, Preliminaries]).

Let $X$ be a complex Banach space and $a=\left(a_{1}, \ldots, a_{n}\right)$ be a commuting system of linear continuous operators on $X$. Denote by $\operatorname{sp}(a, X)$ the Taylor spectrum of $a$ on $X[6]$.

Received by the editors May 17, 1977.

AMS (MOS) subject classifications (1970). Primary 47D99; Secondary 32C30, 32C35.

Key words and phrases. Commuting system of operators, spectrum, local spectrum, differential form, cohomology, spectral hull, Stokes' theorem.

서 American Mathematical Society 1978 
Let $U$ be an open neighbourhood of $\operatorname{sp}(a, X)$ and $f$ be an $X$-valued analytic function on $U$. Denote by $\mathscr{B}(U, X)$ the space of all continuous $X$-valued functions on $U$ which are infinitely differentiable, in the sense of distributions, with respect to $\bar{z}_{1}, \ldots, \bar{z}_{n}[7, \S 2]$. Consider a system $\sigma=$ $\left(s_{1}, \ldots, s_{n}\right)$ of indeterminates and denote by $\Lambda^{p}[\sigma \cup d \bar{z}, \mathscr{B}(U, X)]$ the space of all forms of degree $p$ in the indeterminates $s_{1}, \ldots, s_{n}, d \bar{z}_{1}, \ldots, d \bar{z}_{n}$ having the coefficients in $\mathscr{B}(U, X)$. Denote by $\alpha \oplus \bar{\partial}$ the (coboundary) operator defined by

$$
\begin{aligned}
{[(\alpha \oplus \bar{\partial}) \psi](z)=} & {\left[\left(z_{1}-a_{1}\right) s_{1}+\cdots+\left(z_{n}-a_{n}\right) s_{n}\right.} \\
& \left.+\left(\partial / \partial \bar{z}_{1}\right) d \bar{z}_{1}+\cdots+\left(\partial / \partial \bar{z}_{n}\right) d \bar{z}_{n}\right] \wedge \psi(z), \quad z \in U,
\end{aligned}
$$

where $\psi \in \Lambda^{p}[\sigma \cup d \bar{z}, \mathscr{B}(U, X)]$.

The Cauchy-Weil integral of $f$ with respect to $a$ (on $U$ ) is an element of $X$ obtained as follows. Since $U$ is an open neighbourhood of $\operatorname{sp}(a, X)$, the cohomology class of the form of $s f=f s_{1} \wedge \cdots \wedge s_{n}$, with respect to $\alpha \oplus \bar{\partial}$, contains a form $\chi$ with compact support (i.e. there exist a form $\chi$ with compact support and another form $\psi$ such that $s f-\chi=(\alpha \oplus \bar{\partial}) \psi)$. Denote by $\pi \chi$ that part of $\chi$ containing only the indeterminates $d \bar{z}_{1}, \ldots, d \bar{z}_{n}$. That will be of the form $\pi \chi=h d \bar{z}_{1} \wedge \cdots \wedge d \bar{z}_{n}$, where $h \in \mathscr{B}(U, X)$ and $h$ has compact support.

The Cauchy-Weil integral of $f$ with respect to $a$ (on $U$ ) is defined by

$$
\begin{aligned}
\int_{U} R_{z-a} f(z) & \wedge d z_{1} \wedge \cdots \wedge d z_{n} \\
=\int_{U}(-1)^{n} \pi \chi & \wedge d z_{1} \wedge \cdots \wedge d z_{n} .
\end{aligned}
$$

The value of the integral on the right side depends only on the cohomology class of $\chi$ (as form with compact support) with respect to $\alpha \oplus \bar{\partial}$. Moreover, it does not change if $U$ becomes smaller and depends continuously on the function $f$.

With the Cauchy-Weil integral at hand, it is easy to define the functional analytic calculus. If $f$ is a scalar function analytic in an open neighbourhood of $\operatorname{sp}(a, X)$, then $[7, \S 4] f(a)$ is defined by

$$
f(a) x=\frac{1}{(2 \pi i)^{n}} \int_{U} R_{z-a} f(z) x \wedge d z_{1} \wedge \cdots \wedge d z_{n}, \quad x \in X .
$$

In particular, taking into account that $1(a)=\mathrm{id}$, we obtain

$$
x=\frac{1}{(2 \pi i)^{n}} \int_{U} R_{z-a} x \wedge d z_{1} \wedge \cdots \wedge d z_{n}, \quad x \in X .
$$

2. The relation between $\sigma(a, x)$ and $\operatorname{sp}(a, x)$. We may now define the local spectrum $\operatorname{sp}(a, x)$ as the support of the integrand from (3), having in view to obtain a local variant of this formula.

DEFINITION 1 [4]. The resolvent set $r(a, x)$ of $x$ with respect to $a$ is the union 
of all open sets $D$ with the property that there exists a form $\psi \in \Lambda^{n-1}[\sigma \cup d \bar{z}$, $\mathscr{B}(D, X)]$, satisfying $s x=(\alpha \oplus \bar{\partial}) \psi$. The spectrum $\operatorname{sp}(a, x)$ of $x$ with respect to $a$ is the complement in $C^{n}$ of $r(a, x), \operatorname{sp}(a, x)=C^{n} \backslash r(a, x)$.

If $n=1$ then the form $\psi$ in Definition 1 is of degree zero and, consequently, it is simply a function from $\mathscr{B}(D, X)$. Moreover, the relation $s x=(\alpha \oplus \bar{\partial}) \psi$ is equivalent to $x=(z-a) \psi(z), z \in D$ and $(\partial / \partial \bar{z}) \psi=0$. Therefore $\psi$ is an analytic function on $D$ and, consequently, we have, in this case, $\operatorname{sp}(a, x)=\sigma(a, x)$.

If the equation in $\psi, s x=(\alpha \oplus \bar{\partial}) \psi$, has a global solution defined on $r(a, x)$ then, for any open neighbourhood $V$ of $\operatorname{sp}(a, x)$, the cohomology class of $s x$ with respect to $\alpha \oplus \bar{\partial}$ contains a form $\chi$ with compact support in $V$ [4, $\S 1]$ and, consequently, we get the following local variant of (3):

$$
x=\frac{1}{(2 \pi i)^{n}} \int_{V}(-1)^{n} \pi \chi \wedge d z_{1} \wedge \cdots \wedge d z_{n} .
$$

Just this local variant of (3) motivates our Definition 1 . It is easy to see that, in the case $n=1$, the equation $s x=(\alpha \oplus \bar{\partial}) \psi$ has a global solution if and only if the operator $a$ has the single-valued extension property [3], [2].

DEFINITION 2. The system $a$ is said to have the localization property (in short, property $\mathrm{L})$ if $H^{n-1}(\mathscr{B}(G, X), \alpha \oplus \bar{\partial})=0$ for any open set $G \subset C^{n}$.

We have denoted by $H^{n-1}(\mathscr{B}(G, X), \alpha \oplus \bar{\partial})$ the cohomology module of order $n-1$ for the cochain complex consisting of the spaces $\Lambda^{p}[\sigma \cup d \bar{z}$, $\mathscr{B}(G, X)]$ and of $\alpha \oplus \bar{\partial}$ as coboundary operator.

Let $U$ be an open set in $C^{n}$ and let us denote by $\mathscr{U}(U, X)$ the space of all $X$-valued analytic functions on $U$. Denote by $\alpha$ the operator defined by

$$
(\alpha \psi)(z)=\left[\left(z_{1}-a_{1}\right) s_{1}+\cdots+\left(z_{n}-a_{n}\right) s_{n}\right] \wedge \psi(z), \quad z \in U,
$$

where $\psi$ is an exterior form in $s$ with coefficients in $\mathcal{Q}(U, X)$. Then the object consisting of the spaces $\Lambda^{p}[\sigma, \mathcal{Q}(U, X)]$ and of $\alpha$ as coboundary operator is a cochain complex. We shall denote by $H^{i}(U(U, X), \alpha)$ the cohomology modules of this complex.

DEFINITION 3. The system $a$ is said to have the analytic localization property (in short property LA) if $H^{i}(\mathscr{U}(U, X), \alpha)=0,0 \leqslant i \leqslant n-1$, for any open polydisc $U \subset C^{n}$.

We have proved in [4] that property LA implies property L. If $n=1$ then the properties are equivalent to the single-valued extension property.

Definition 4 [7]. Let $K \subset C^{n}$ be a compact set. The spectral hull of $K$ is the set of all elements $w=\left(w_{1}, \ldots, w_{n}\right) \in C^{n}$ with the property that the equation

$$
\left(z_{1}-w_{1}\right) f_{1}(z)+\cdots+\left(z_{n}-w_{n}\right) f_{n}(z)=1
$$

has no analytic solutions $f_{1}, \ldots, f_{n}$ in any open neighbourhood of $K$.

We shall prove that, for any $x \in X, \sigma(a, x)$ is contained in the spectral hull of $\operatorname{sp}(a, x)$. For that we need two lemmas.

LemMA 1. Let $K$ be a compact subset of $C^{n}$. If $w=\left(w_{1}, \ldots, w_{n}\right)$ does not belong to the spectral hull of $K$ then there exist an open neighbourhood $U$ of $K$, 
an open neighbourhood $V$ of $w$ and $n$ scalar functions $f_{1}(z, \zeta), \ldots, f_{n}(z, \zeta)$ analytic on $U \times V$, satisfying

$$
\sum_{j=1}^{n}\left(z_{j}-\zeta_{j}\right) f_{j}(z, \zeta) \equiv 1, \quad(z, \zeta) \in U \times V .
$$

Proof. Since $w$ does not belong to the spectral hull of $K$, it follows, according to Definition 4, that there exist an open neighbourhood $U_{1}$ of $K$ and $n$ scalar analytic functions $f_{1}, \ldots, f_{n}$ on $U_{1}$, such that

$$
\sum_{j=1}^{n}\left(z_{j}-w_{j}\right) f_{j}(z) \equiv 1, \quad z \in U_{1}
$$

From here we obtain

$$
\begin{aligned}
\sum_{j=1}^{n}\left(z_{j}-\zeta_{j}\right) f_{j}(z) & =\sum_{j=1}^{n}\left(z_{j}-w_{j}\right) f_{j}(z)+\sum_{j=1}^{n}\left(w_{j}-\zeta_{j}\right) f_{j}(z) \\
& =1+\sum_{j=1}^{n}\left(w_{j}-\zeta_{j}\right) f_{j}(z), \quad z \in U_{1} .
\end{aligned}
$$

Let $U$ be an open relatively compact neighbourhood of $K$ such that $\bar{U} \subset U_{1}$. It is easy to see that there exists an open neighbourhood $V$ of $w$ such that $\left|\sum_{j=1}^{n}\left(w_{j}-\zeta_{j}\right) f_{j}(z)\right|<1$ for any $\zeta \in V$ and any $z \in U$. Thus, for any $(z, \zeta)$ $\in U \times V$, the complex number

$$
\sum_{j=1}^{n}\left(z_{j}-\zeta_{j}\right) f_{j}(z)=1+\sum_{j=1}^{n}\left(w_{j}-\zeta_{j}\right) f_{j}(z)
$$

is different from zero. Denoting

$$
h(z, \zeta)=\left[\sum_{j=1}^{n}\left(z_{j}-\zeta_{j}\right) f_{j}(z)\right]^{-1}, \quad(z, \zeta) \in U \times V,
$$

we shall obtain an analytic function on $U \times V$ satisfying

$$
h(z, \zeta)\left[\sum_{j=1}^{n}\left(z_{j}-\zeta_{j}\right) f_{j}(z)\right] \equiv 1, \quad(z, \zeta) \in U \times V .
$$

Therefore we may define

$$
f_{j}(z, \zeta)=h(z, \zeta) f_{j}(z), \quad(z, \zeta) \in U \times V, 1 \leqslant j \leqslant n,
$$

and the statement of Lemma 1 follows.

Lemma 2. Suppose that the system a satisfies L. Let $U$ be an open neighbourhood of the set $\operatorname{sp}(a, x)$ and $f$ a scalar analytic function on $U$. Then, for any form appearing in $\left(3^{\prime}\right)$ and any number $j, 1 \leqslant j \leqslant n$, we have

$$
\int_{U}(-1)^{n} f(z)\left(z_{j}-a_{j}\right) \pi \chi \wedge d z_{1} \wedge \cdots \wedge d z_{n}=0
$$

Proof. We shall apply the theorem of Stokes. Let $j$ be a fixed number, $1 \leqslant j \leqslant n$, and let us denote by $\tilde{a}_{j}$ the operator defined by $\left(\tilde{a}_{j} \psi\right)(z)=\left(z_{j}-\right.$ $\left.a_{j}\right) \psi(z), z \in U$, where $\psi$ is a form in $s$ and $d \bar{z}$ having coefficients in $\mathscr{B}(U, X)$. 
Let $\chi$ be an arbitrary form from those appearing in (3'). Then there exists a form $\psi^{*}$ on $U$ such that $s x-\chi=(\alpha \oplus \bar{\partial}) \psi^{*}$. By applying the operator $\tilde{a}_{j}$ to both sides of this equality and multiplying by $f$, we obtain

$$
s f \tilde{a}_{j} x-f \tilde{a}_{j} \chi=(\alpha \oplus \bar{\partial})\left(f \tilde{a}_{j} \psi^{*}\right) .
$$

On the other hand, denoting

$$
\tilde{\psi}_{j}(z)=f(z) x s_{1} \wedge \cdots \wedge \hat{s}_{j} \wedge \cdots \wedge s_{n}, \quad z \in U,
$$

we have $s f \tilde{a}_{j} x=(\alpha \oplus \bar{\partial}) \tilde{\psi}_{j}$, whence

$$
f \tilde{a}_{j} \chi=(\alpha \oplus \bar{\partial})\left(\tilde{\psi}_{j}-f \tilde{a}_{j} \psi^{*}\right) .
$$

Taking into account that $\chi$ has compact support in $U$, it follows that $f \tilde{a}_{j} \chi$ has compact support in $U$. By using property $\mathrm{L}$ we shall prove that $f \tilde{a}_{j} \chi=(\alpha$ $\oplus \bar{\partial}) \psi_{j}$ on $U$, where $\psi_{j}$ has also compact support. With this result at hand, we get

$$
f \tilde{a}_{j} \pi \chi=\pi\left(f \tilde{a}_{j} \chi\right)=\pi(\alpha \oplus \bar{\partial}) \psi_{j}=\bar{\partial}\left(\pi \psi_{j}\right) .
$$

From here we obtain

$$
\begin{array}{rl}
\int_{U}(-1)^{n} & f(z)\left(z_{j}-a_{j}\right) \pi \chi \wedge d z_{1} \wedge \cdots \wedge d z_{n} \\
& =\int_{U}(-1)^{n} f \tilde{a}_{j} \pi \chi \wedge d z_{1} \wedge \cdots \wedge d z_{n} \\
& =\int_{U}(-1)^{n} \bar{\partial}\left(\pi \psi_{j}\right) \wedge d z_{1} \wedge \cdots \wedge d z_{n} \\
& =\int_{U}(-1)^{n} d\left(\pi \psi_{j} \wedge d z_{1} \wedge \cdots \wedge d z_{n}\right) .
\end{array}
$$

Taking into account that $\psi_{j}$ has compact support in $U$ and applying the theorem of Stokes, we deduce that the last integral is equal to zero and, consequently,

$$
\int_{U}(-1)^{n} f(z)\left(z_{j}-a_{j}\right) \pi \chi \wedge d z_{1} \wedge \cdots \wedge d z_{n}=0
$$

Therefore it remains to prove that there exists a form $\psi_{j}$ with compact support such that $f \tilde{a}_{j} \chi=(\alpha \oplus \bar{\partial}) \psi_{j}$. Denote by $K$ the support of $\chi$ (which is a compact set). Since $f \tilde{a}_{j} \chi=(\alpha \oplus \partial)\left(\tilde{\psi}_{j}-f \tilde{a}_{j} \psi^{*}\right)$ and $\chi=0$ on $U \backslash K$, we have $(\alpha \oplus \bar{\partial})\left(\tilde{\psi}_{j}-f \tilde{a}_{j} \psi^{*}\right)=0$ on $U \backslash K$. Thus, by applying property $L$ for $G=U \backslash$ $K$, there exists a form $\varphi_{j}$ such that $\tilde{\psi}_{j}-f \tilde{f}_{j} \psi^{*}=(\alpha \oplus \bar{\partial}) \varphi_{j}$. Consider now two open relatively compact sets $U_{1}$ and $U_{2}$ such that $K \subset U_{1} \subset \bar{U}_{1} \subset U_{2} \subset \bar{U}_{2}$ $\subset U$. Let $h$ be a $C^{\infty}$ scalar function on $U$ such that $h=0$ on $U_{1}$ and $h=1$ on $U \backslash U_{2}$. Let us define the form $\tilde{\varphi}_{j}$ by

$$
\begin{aligned}
\tilde{\varphi}_{j} & =h \varphi_{j} \quad \text { on } U \backslash K, \\
& =0 \text { on } U_{1} .
\end{aligned}
$$

Denoting $\psi_{j}=\left(\tilde{\psi}_{j}-f \tilde{a}_{j} \psi^{*}\right)-(\alpha \oplus \bar{\partial}) \tilde{\varphi}_{j}$, we obtain 


$$
(\alpha \oplus \bar{\partial}) \psi_{j}=(\alpha \oplus \bar{\partial})\left(\tilde{\psi}_{j}-f \tilde{a}_{j} \psi^{*}\right)=f \tilde{a}_{j} \chi .
$$

On the other hand, we have $\tilde{\varphi}_{j}=h \varphi_{j}$ on $U \backslash K$ and $h=1$ on $U \backslash U_{2}$, whence $\psi_{j}=0$ on $U \backslash U_{2}$. This completes the proof.

We can prove now our main result concerning the relation between the spectrum $\sigma(a, x)$ defined in the Introduction and the spectrum $\operatorname{sp}(a, x)$.

THEOREM. Let a be a commuting system of operators satisfying property $\mathrm{L}$. Then, for any $x \in X$, the analytic spectrum $\sigma(a, x)$ is contained in the spectral hull of the spectrum $\operatorname{sp}(a, x)$.

Proof. We shall prove that if $w$ does not belong to the spectral hull of $\operatorname{sp}(a, x)$ then $w \in \rho(a, x)$, from where the Theorem will obviously follow. According to Lemma 1, there exist an open neighbourhood $U$ of $\operatorname{sp}(a, x)$, an open neighbourhood $V$ of $w$ and $n$ scalar functions $f_{1}(z, \zeta), \ldots, f_{n}(z, \zeta)$, analytic on $U \times V$ and satisfying

$$
\sum_{j=1}^{n}\left(z_{j}-\zeta_{j}\right) f_{j}(z, \zeta) \equiv 1, \quad(z, \zeta) \in U \times V .
$$

Let us define the $X$-valued functions $f_{j}$ on $V$ by

$$
f_{j}(\zeta)=-\frac{1}{(2 \pi i)^{n}} \int_{U}(-1)^{n} f_{j}(z, \zeta) \pi \chi \wedge d z_{1} \wedge \cdots \wedge d z_{n}, \quad 1 \leqslant j \leqslant n,
$$

where $\chi$ is one of the forms appearing in $\left(3^{\prime}\right)$. Since the form $\chi$ has compact support, the forms appearing under the integral also have compact support. Moreover, since $f_{j}(z, \zeta)$ are analytic functions, $1 \leqslant j \leqslant n$, it is easy to see that $f_{j}$ are analytic functions on $V, 1 \leqslant j \leqslant n$. We shall prove that $\sum_{j=1}^{n}\left(\zeta_{j}-\right.$ $\left.a_{j}\right) f_{j}(\zeta) \equiv x, \zeta \in V$, and thus it will follow that $w \in \rho(a, x)$, as desired.

By applying Lemma 2 for the functions $f_{j}(z, \zeta)$, we get

$$
\int_{U}(-1)^{n} f_{j}(z, \zeta)\left(z_{j}-a_{j}\right) \pi \chi \wedge d z_{1} \wedge \cdots \wedge d z_{n}=0, \quad 1 \leqslant j \leqslant n .
$$

Consequently, we have

$$
\begin{aligned}
& \sum_{j=1}^{n}\left(\zeta_{j}-a_{j}\right) f_{j}(\zeta) \\
&=-\frac{1}{(2 \pi i)^{n}} \int_{U}(-1)^{n} \sum_{j=1}^{n}\left(\zeta_{j}-a_{j}\right) f_{j}(z, \zeta) \pi \chi \wedge d z_{1} \wedge \cdots \wedge d z_{n} \\
&=-\frac{1}{(2 \pi i)^{n}} \int_{U}(-1)^{n} \sum_{j=1}^{n}\left(\zeta_{j}-z_{j}\right) f_{j}(z, \zeta) \pi \chi \wedge d z_{1} \wedge \cdots \wedge d z_{n} \\
&-\frac{1}{(2 \pi i)^{n}} \int_{U}(-1)^{n} \sum_{j=1}^{n}\left(z_{j}-a_{j}\right) f_{j}(z, \zeta) \pi \chi \wedge d z_{1} \wedge \cdots \wedge d z_{n} \\
&= \frac{1}{(2 \pi i)^{n}} \int_{U}(-1)^{n} \pi \chi \wedge d z_{1} \wedge \cdots \wedge d z_{n}=x .
\end{aligned}
$$

For the last equality we have used (3'). Therefore 


$$
\sum_{j=1}^{n}\left(\zeta_{j}-a_{j}\right) f_{j}(\zeta) \equiv x, \quad \zeta \in V,
$$

and the proof is finished.

REMARK. It is possible to define a local spectrum similar to $\operatorname{sp}(a, x)$ by using the space $C^{\infty}$ instead of the space $\mathscr{B}$. It is clear that $\operatorname{sp}(a, x)$ will be contained in this spectrum. All the results proved in [4] for $\operatorname{sp}(a, x)$ also remain valid for this new spectrum. Indeed, the properties of the space $\mathscr{B}$ used in [4] are still valid for the space $C^{\infty}$ (see also [5]).

\section{REFERENCES}

1. E. Albrecht, Funktionalkalküle in mehreren veränderlichen, Ph.D. Dissertation, Mainz Univ., 1972.

2. I. Colojoară and C. Foias, Theory of generalized spectral operators, Gordon and Breach, New York, 1968.

3. N. Dunford, Spectral operators, Pacific J. Math. 4 (1954), 321-354.

4. S, Frunză, The Taylor spectrum and spectral decompositions, J. Functional Analysis 19 (1975), $390-421$.

5. __ On Taylor functional calculus, Studia Math. 52 (1974), 239-242.

6. J. L. Taylor, A joint spectrum for several commuting operators, J. Functional Analysis 6 (1970), 172-191.

7. _ Analytic functional calculus for several commuting operators, Acta Math. 125 (1970), 1-38.

Department of Mathematics, University of IASI, IASI, Romînia 